\title{
Utilization of combined PD-L1 expression and neutrophil-to- lymphocyte ratio prior to surgery as a prognostic factor in non- small cell lung cancer with brain metastasis
}

\author{
Lei Xia ${ }^{1,2}$, Hu Huang ${ }^{1,3}, \mathrm{He} \mathrm{Xiao}^{1}$, Dong Wang ${ }^{1}$, Zhenzhou Yang $^{1,2}$ \\ ${ }^{1}$ Cancer Center, Daping Hospital and Research Institute of Surgery, Army Medical University, Chongqing 400042, China; ${ }^{2}$ Cancer Center, The \\ Second Affiliated Hospital of Chongqing Medical University, Chongqing 400000, China; ${ }^{3}$ Department of Oncology, The 161 Hospital of PLA, \\ Wuhan 430010, China \\ Contributions: (I) Conception and design: Z Yang; (II) Administrative support: D Wang; (III) Provision of study materials or patients: L Xia; (IV) \\ Collection and assembly of data: H Huang; (V) Data analysis and interpretation: H Xiao; (VI) Manuscript writing: All authors; (VII) Final approval \\ of manuscript: All authors. \\ Correspondence to: Professor Zhenzhou Yang. Cancer Center, The Second Affiliated Hospital of Chongqing Medical University, No. 288 Tianwen \\ Road, Nan'an District, Chongqing 400000, China. Email: yangzz1970@163.com.
}

Background: This study was undertaken to investigate the expression of programmed death-ligand 1 (PDL1) in the pulmonary primary tumor and brain metastases (BM) tumor as well as neutrophil-to-lymphocyte ratio (NLR) in preoperative peripheral blood samples in advanced non-small cell lung cancer. The potential association between both PD-L1 expression and NLR value and therapeutic sensitivity along with prognosis was explored.

Methods: Eleven patients pathologically confirmed as non-small cell lung cancer (NSCLC) with BM were enrolled. The expression of PD-L1 in pulmonary primary tumor and BM tumor was evaluated with immunohistochemistry. NLR value was calculated from blood cell count. DFS and OS were calculated using the Kaplan-Meier method, and the log-rank test was used to assess differences between groups.

Results: High expression of PD-L1 was observed in 4 pulmonary primary tumors and 3 BM tumors respectively. The NLR in peripheral blood before brain surgery was shown to be efficient to discriminate patients whose brain metastatic lesion reached disease control from others (AUC: 0.917 95\% CI: 0.731$1.000, \mathrm{P}=0.041$ ). Five patients had an NLR value higher than the cutoff value of 3.0553 . Eight patients with low expression of PD-L1 in BM lesions had 27 months of median OS, which was signifcantly longer than patients with high expression of PD-L1 with 10 months of median OS (Log-Rank, P=0.012). Five patients with both low expression of PD-L1 in BM lesions and low NLR value $(\leq 3.0553)$ reached 36 months mOS significantly longer than others with only 10 months of mOS (Log-Rank, $\mathrm{P}=0.034, \mathrm{HR}=0.133$, 95\% CI: 0.861-65.819, $\mathrm{P}=0.068)$.

Conclusions: High expression of PD-L1 along with high NLR value is associated with less sensitivity of response to treatment and poor prognosis.

Keywords: Programmed death-ligand 1 (PD-L1); neutrophil-to-lymphocyte ratio (NLR); non-small cell lung cancer (NSCLC); brain metastases (BM); prognosis

Submitted Jul 23, 2019. Accepted for publication Oct 25, 2019.

doi: $10.21037 /$ tcr.2019.11.08

View this article at: http://dx.doi.org/10.21037/tcr.2019.11.08 


\section{Introduction}

Brain metastases $(\mathrm{BM})$ are found about $23-65 \%$ of patients with lung cancer, constituting around half of all brain metastasis (1). Improved diagnosis and therapy for nonsmall cell lung cancer (NSCLC) have increased the diagnosis rate of NSCLC with BM. Once brain metastasis occurs, the prognosis is poor with a survival time of only 2-4 months (2). Although whole-brain radiotherapy (WBRT), stereotactic radiosurgery (SRS), surgical removal of single BM and other methods are used, satisfactory local control and survival remains difficult to achieve as chemotherapy drugs do not pass through the blood-brain barrier, and therefore have limited therapeutic effects on BM. The recent gradual improvements in comprehensive NSCLC treatment have resulted in clinical oncology research focusing on improving survival and quality of life for patients with NSCLC with BM.

Programmed cell death ligand 1 (PD-L1) also known as cluster of differentiation 274 (CD274) or B7 homolog 1 (B7-H1) is encoded by gene CD274 and belongs to type I transmembrane protein with molecular weight $40 \mathrm{kDa}$ (3). Under the normal physiological circumstance, the human immune system is enabled to sense exogenous antigens accumulated in node or spleen and induce proliferation of cytotoxic killer T lymphocyte (CD8+ T lymphocyte). However, binding of PD-L1 to PD-1 leads to initiate inhibitory signaling to decrease proliferation of CD8+ T lymphocyte. Meanwhile, PD-1 can control aggregation of antigen-specific T cells through regulation of Bcl-2 gene (4).

The overexpression of PD-L1 has been observed in different kind of cancers including non-small cell lung cancer $(5,6)$. Both preclinical researches and meta-analysis suggested that overexpression of $\mathrm{PD}-\mathrm{L} 1$ is associated with worse prognosis and shorter overall survival (OS) (7-10). Recently, several clinical trials have been carried out to evaluate efficiency and safety of immune checkpoint inhibitors against PD-/PD-L1 in various solid tumors and revealed encouraging results. However, not all patients uniformly response to immune checkpoint inhibitors and few biomarkers including immunohistochemical test of PD-L1 have become routinely used in clinical practice for selection of patients potentially benefited from immune checkpoint therapy. Furthermore, the effectiveness of currently used biomarkers is still limited and other accompanied biomarkers are eagerly needed.

Growing evidence revealed that inflammatory response to cancerous cells is associated with antitumor immune response in the tumor microenvironment (11). Amongst prognostic evaluation systems based on inflammatory response is the neutrophil-to-lymphocyte ratio (NLR) which has greatly attracted attention (12-14). Several studies have suggested that high NLR is closely relevant to worse prognosis in different cancers such as gastric cancer, non-small cell lung cancer and colorectal cancer (15-17). A retrospective study comprised of 58 cases with advanced non-small cell lung cancer revealed that the patients with NLR $\geq 4$ before treatment exhibit shorter DFS and OS compared those with NLR <4 (DFS: 10.1 vs. 11.4 months; OS: $19.5 v s$. NS months). This result provided evidence that NLR could be utilized as a predictive factor for PD-1/PDL1 immune checkpoint therapy.

Few researches have been undertaken to explore the consistency of PD-L1 expression level between lung primary lesion and brain metastatic lesions and association between PD-L1 expression level and NLR. More importantly, the predictive efficiency of the combination of PD-L1 expression and NLR are still not evaluated up to now in advanced NSCLC. Our current study presents the status of PD-L1 expression in paired lung primary lesions and brain metastatic lesions and NLR in peripheral blood in 11 patients with advanced NSCLC underwent surgery to uncover the relationship between NLR and PDL1 expression and their capacity as prognostic biomarkers for improvement of life quality and survival for advanced NSCLC with brain metastasis.

\section{Methods}

\section{Patients}

Eleven patients treated at Daping Hospital and Research Institute of Surgery (Chongqing, China) for the period between January 2010 and January 2015, inclusive, provided clinical data. The patients had PPT and BM that had been surgically removed and pathologically confirmed as NSCLC with BM. Ages ranged from 26-72 years with a median age of 58 . There were 3 females $(27.3 \%)$ and 8 males $(72.7 \%)$. Two patients had squamous carcinoma (18.2\%) and 9 patients had adenocarcinoma (81.8\%). This study was conducted rigorously according to the Declaration of Helsinki and the principle of International Ethical Guidelines for Biomedical Research Involving Human constituted by the World Health Organization (WHO) and the Council for International Organizations of Medical Sciences (CIOMS) and was approved by the Ethics Committee of the Daping Hospital. The written consensus 
was obtained from all participants.

Patient information including age, sex, height, weight, smoking history and family history; disease information including histological type, clinical stage $(18,19)$, Eastern Cooperative Oncology Group (ECOG) performance status (PS) score and surgery history; initial treatment details including chemotherapy regimen and cycles, radiotherapy regimen and cycles, efficacy evaluation and side effects; and follow-up information including the follow-up period, time of the last follow-up, disease-free survival (DFS) and OS of patients with pulmonary and cerebral disease, was obtained from clinical records and questionnaires. Smoking history was defined as follows: never smoking, those who had smoked $\leq 100$ cigarettes and those who had never smoked; former smokers, those who had smoked $>100$ cigarettes in total and quit smoking $\geq 1$ year previously; present smoker, those who had smoked $>100$ cigarettes in total and quit $\leq 1$ year previously or current smokers.

\section{Expression of PD-L1 in PPT and BM detected by immunobistochemistry}

Patient PPT and BM specimens were obtained via complete tumor resection, fixed with $10 \%$ formalin, embedded in paraffin and divided into $4-\mu \mathrm{m}$ sections. 11 matched pairs of PPT and BM specimens from the same patients were subjected to immunohistochemistry analysis. The working dilution of Rabbit anti-human PD-L1 monoclonal antibody (ab213524) was 1:100. PBS was used as a negative control. An EnVision two-step method (\#33535.GB/T16494-1996; Agilent Technologies, Inc., Santa Clara, CA, USA) was used according to the manufacturer's protocol. Continuous 4- $\mu \mathrm{m}$ paraffin sections were deparaffinized with xylene, hydrated by ethanol gradients, and incubated with $3 \% \mathrm{H}_{2} \mathrm{O}_{2}$ in methanol at room temperature for $10 \mathrm{~min}$. Antigen retrieval method was performed by microwave heating. The sections were incubated with the primary antibodies overnight at $4{ }^{\circ} \mathrm{C}$. Then $50 \mu \mathrm{L}$ of compound [HRP conjugated antirabbit/mouse IgG goat antibody (working solution), KIT-5020, 160415407F] was added. The sections were then incubated at $37^{\circ} \mathrm{C}$ for $30 \mathrm{~min}$, developed using diaminobenzidine- $\mathrm{H}_{2} \mathrm{O}_{2}$ and stained with hematoxylin. PBS was used as a negative control.

\section{Determination of immunobistochemical analysis of PD-L1 expression}

A positive PD-L1 expression signal was determined as brown-yellow fine granules. Five representative fluorescence microscopy images of various fields of view (magnification, $\times 400$ ) were randomly selected for each slice. The positive region was selected using the automatic image analysis system in Image-pro Plus 5.1 software (Media Cybernetics, Inc., Rockville, MD, USA), which calculated the integral absorbance values to evaluate $\mathrm{PD}-\mathrm{L} 1$ protein expression levels, in which - and + were recorded as low expression, whereas ++ was recorded as high expression. Cancer cells with a cytoplasm or nucleus stained at $>10 \%$ were deemed to have positive cytoplasmic or nuclear expression of cytoplasm. Cytoplasmic expression, or nucleoplasm co-expression, is referred to as "shift-from-nucleus-tocytoplasm".

\section{$N L R$ value determination before surgery}

Peripheral venous blood samples were collected before surgery. The neutrophils and lymphocytes count was obtained from routine blood examination and NLR value was calculated from count values. Receiver operating characteristic (ROC) curve analysis was used to select optimal cutoff value based on Youden index.

\section{Chemotherapy and radiotherapy regimens}

Patients received a platinum-containing doublet regimen as follows: TP regimen, Taxol $\left(135 \mathrm{mg} / \mathrm{m}^{2}\right.$, day 1$)$ plus cisplatin $\left(75 \mathrm{mg} / \mathrm{m}^{2}\right.$, day 1$)$; DP regimen, docetaxel $\left(75 \mathrm{mg} / \mathrm{m}^{2}\right.$, day 1) plus cisplatin $\left(75 \mathrm{mg} / \mathrm{m}^{2}\right.$, day 1$)$. The targeted treatment regimen was as follows: Erlotinib hydrochloride tablets, $150 \mathrm{mg} / \mathrm{day}$, once a day; temozolomide capsules, $75 \mathrm{mg} / \mathrm{m}^{2}$ per day, during radiotherapy. The patient radiotherapy regimen was as follows: two irradiation fields (DT40 Gy/20 f/4 w) and SRS.

\section{Prognosis and therapeutic effect evaluation}

The main outcome measures were DFS and OS. DFS was defined as the time post-medication or treatment to recurrence, whereas OS was the time from treatment start to mortality or to the date of last follow-up.

Secondary outcome measures were objective response rate (ORR) and disease control rate (DCR). The therapeutic effect evaluation criteria were whether local disease progressed following surgery and were detected by computed tomography, magnetic resonance imaging or other imaging methods to target lesions or metastases 
following conventional treatment. The therapeutic effect was evaluated according to Response Evaluation Criteria in Solid Tumors, version 1.1 (20), which includes complete response (CR), partial response (PR), stable disease (SD) and progressive disease (PD). ORR was defined as the sum of CR and PR. DCR was defined as the sum of CR, PR, and SD.

\section{Follow-up}

Patients received follow up every month in the first six months and every 2 months thereafter until mortality. Quality of life, weight loss, and other indicators were evaluated. The follow-up deadline was December 2016. No patients were lost to follow-up.

\section{Statistical analysis}

All the material and data were processed according to the same standard prior to statistical analysis, input into Windows Excel (Microsoft Corporation, Redmond, WA, USA) tables and checked several times. Code data were generated according to a statistical analysis format. All data were analyzed using software package SPSS 19.0 (IBM SPSS, Armonk, NY, USA). ORR, DCR and side effects were compared using the Pearson $\chi^{2}$ test and Fisher's exact test. Median DFS and OS and corresponding 95\% confidence intervals were evaluated with KaplanMeier estimate with differences between each factor level compared with the log-rank test. Analysis of factors associated with prognosis was performed using the Cox proportional hazards model and hazard ratios. A 95\% confidence interval for each factor was calculated. ROC curve analysis was performed to assess the discriminative ability of NLR prior brain surgery for disease control of brain metastatic lesions. Under the significance level $\alpha=0.05$, a two-tailed $\mathrm{P}<0.05$ was considered to indicate a statistically significant difference.

\section{Results}

\section{Clinical data and clinical outcomes of patients}

PPT and BM from 11 patients with NSCLC were surgically removed. There were 8 males $(72.7 \%)$ and 3 females (27.3\%). The median age was 58 (range, $26-72$ years). Two patients had squamous carcinoma (18.2\%) and 9 patients had adenocarcinoma (81.8\%). The initial diagnosis staging was: 1 patient (9.1\%) IIIA stage; 4 patients (36.4\%) IIIB stage; 6 patients (54.5\%) IV stage with an ECOG score $\leq 2$. There were 5 patients (45.4\%) who had never smoked, 3 former smokers (27.3\%) and 3 current smokers (27.3\%). There were 4 patients (36.3\%) with BM at the initial diagnosis with the remaining 7 patients $(63.7 \%)$ developing BM during treatment (Table 1).

There were 8 patients $(72.7 \%)$ with an adopted TP regimen, 2 patients $(18.2 \%)$ with erlotinib administered orally and 1 patient $(9.1 \%)$ with temozolomide administered orally. The median chemotherapy cycle number was 4 cycles, one cycle was $28 \mathrm{~d}$, include treatment period and treatment-free period. The treatment period was one day, treatment-free period was 27 days (2-8 cycles). Therapy was performed with WBRT on 2 patients $(18.2 \%)$, SRS in $4(36.3 \%)$ and SRS + WBRT in $1(9.1 \%)$. Four patients $(36.3 \%)$ received no brain lesion radiotherapy. The evaluation of chemotherapeutic effects on pulmonary lesions identified a CR in 3 patients $(27.3 \%)$, PR in 6 $(54.5 \%)$ and SD in $1(9.1 \%)$, whilst 1 patient developed PD $(9.1 \%)$. The response rate to chemotherapy was $81.8 \%$ (Table 1).

The evaluation of WBRT and SRS therapeutic effects on brain lesions identified CR in 3 patients (27.3\%), PR in 4 (36.3\%) and SD in 1 (9.1\%), whilst 3 patients developed PD (27.3\%). The response rate to local treatment was $63.6 \%$.

Follow-up ended Dec 1st, 2016. The median followup time was 20 months (range, 6-38 months). All patients succumbed to disease during follow-up. The median DFSlung was 12 months (95\% CI, 5.934-18.066 months) and median DFSbrain was 7 months (95\% CI, 0.52613.474 months). The median OS was 20 months (95\% CI, 9.045-30.955 months) (Table 1).

\section{Immunobistochemical staining results of PD-L1 in PPT and $B M$}

A PD-L1-positive reaction manifests as brown-yellow granules. Cytoplasm expression was found. There were certain differences in the PD-L1 expression intensity in PPT and BM. In PPT, PD-L1 expression intensities were ++ in 4 patients $(36.4 \%)$ (Figure $1 A),+$ in 4 patient $(36.4 \%)$ (Figure $1 B$ ), and - in 3 patients $(27.3 \%$ ) (Figure 1C). In $\mathrm{BM}, \mathrm{PD}-\mathrm{L} 1$ expression intensities were ++ in 3 patients $(27.3 \%)$ (Figure $1 D),+$ in 4 patient $(36.4 \%$ ) (Figure $1 E$ ) and - in 4 patients (36.4\%) (Figure $1 F$ ). Matched-pair analysis (11 matched pairs of PPT and BM specimens from same patients were subjected to immunohistochemistry analysis) 
Table 1 Clinical characteristics of patients with non-small cell lung cancer

\begin{tabular}{|c|c|}
\hline Patient characteristics & N (\%) \\
\hline Number of patients & 11 \\
\hline Median age & 58 [26-72] \\
\hline \multicolumn{2}{|l|}{ Sex } \\
\hline Male & $8(72.7)$ \\
\hline Female & $3(27.3)$ \\
\hline \multicolumn{2}{|l|}{ ECOG PS } \\
\hline 0 & $1(9.1)$ \\
\hline 1 & $9(81.8)$ \\
\hline 2 & $1(9.1)$ \\
\hline \multicolumn{2}{|l|}{ Tumor stage } \\
\hline IIIA & $1(9.1)$ \\
\hline IIIB & $4(36.4)$ \\
\hline IV & $6(54.5)$ \\
\hline \multicolumn{2}{|l|}{ Histological type } \\
\hline Adenocarcinoma & $9(81.8)$ \\
\hline Squamous cell & $2(18.2)$ \\
\hline \multicolumn{2}{|l|}{ Smoking status } \\
\hline Never & $5(45.5)$ \\
\hline Former smoker & $3(27.3)$ \\
\hline Current smoker & $3(27.3)$ \\
\hline \multicolumn{2}{|l|}{ BM time } \\
\hline Synchronous & $4(36.4)$ \\
\hline Nonsynchronous & 7 (63.6) \\
\hline \multicolumn{2}{|l|}{ Number of BM } \\
\hline Single & $7(63.6)$ \\
\hline Multiple & $4(36.4)$ \\
\hline \multicolumn{2}{|l|}{ Chemotherapy regimens } \\
\hline Platinum-paclitaxel & $8(72.7)$ \\
\hline EGFR-TKI & $2(18.2)$ \\
\hline Temozolomide & $1(9.1)$ \\
\hline \multicolumn{2}{|l|}{ Radiotherapy regimens } \\
\hline No & $4(36.4)$ \\
\hline SRS/WBRT & 7 (63.6) \\
\hline
\end{tabular}

Table 1 (continued)
Table 1 (continued)

\begin{tabular}{ll}
\hline Patient characteristics & $\mathrm{N}(\%)$ \\
\hline Lung response & $3(27.3)$ \\
CR & $6(54.5)$ \\
PR & $1(9.1)$ \\
SD & $1(9.1)$ \\
PD & \\
Brain response & $3(27.3)$ \\
CR & $4(36.4)$ \\
PR & $1(9.1)$ \\
SD & $3(27.3)$ \\
PD &
\end{tabular}

BM, brain metastasis; TKI, tyrosine kinase inhibitors; SRS, stereotactic radiosurgery; WBRT, whole-brain radiotherapy; CR, complete response; PR, partial response; SD, stable disease; $\mathrm{PD}$, progressive disease.

revealed a consistently expressed levels of PD-L1 in PPT and $\mathrm{BM}$ in 6 patients $(54.5 \%)$, which consist of 5 cases with both low expression and one case with both high expressions. Whereas the inconsistently expressed levels of PD-L1 in PPT and BM was observed in 5 cases (45.6\%), in which 3 cases exhibited high expression in PPT and low expression in BM lesions and other two cases had high expression in BT lesions and low in PPT lesions.

\section{ROC curve analysis of $N L R$}

The NLR in peripheral blood prior PPT surgery was $2.97 \pm 1.26$ and NLR prior BM surgery was. The ROC analysis was used to discriminate the patients achieving OR or DC from others based on NLR in peripheral blood prior PPT surgery. However, NLR in peripheral blood prior PPT surgery was completely failed with AUC of 0.778 (95\% CI: $0.490-1.000, P=0.239)$. Meanwhile, NLR between patients achieving OR or DC and those who did not was totally not significant $(\mathrm{P}=0.239$ and $\mathrm{P}=0.752$ respectively, independent-samples Kruskal-Wallis test). So, the NLR in peripheral blood prior PPT surgery was not furtherly employed to analysis. In contrast, NLR prior BM surgery could be able to discriminate the patients achieving OR or DC from others with AUC of 0.917 (95\% CI: 0.731-1.000, $\mathrm{P}=0.041$ ). The cutoff value of 3.0553 based on Youden 

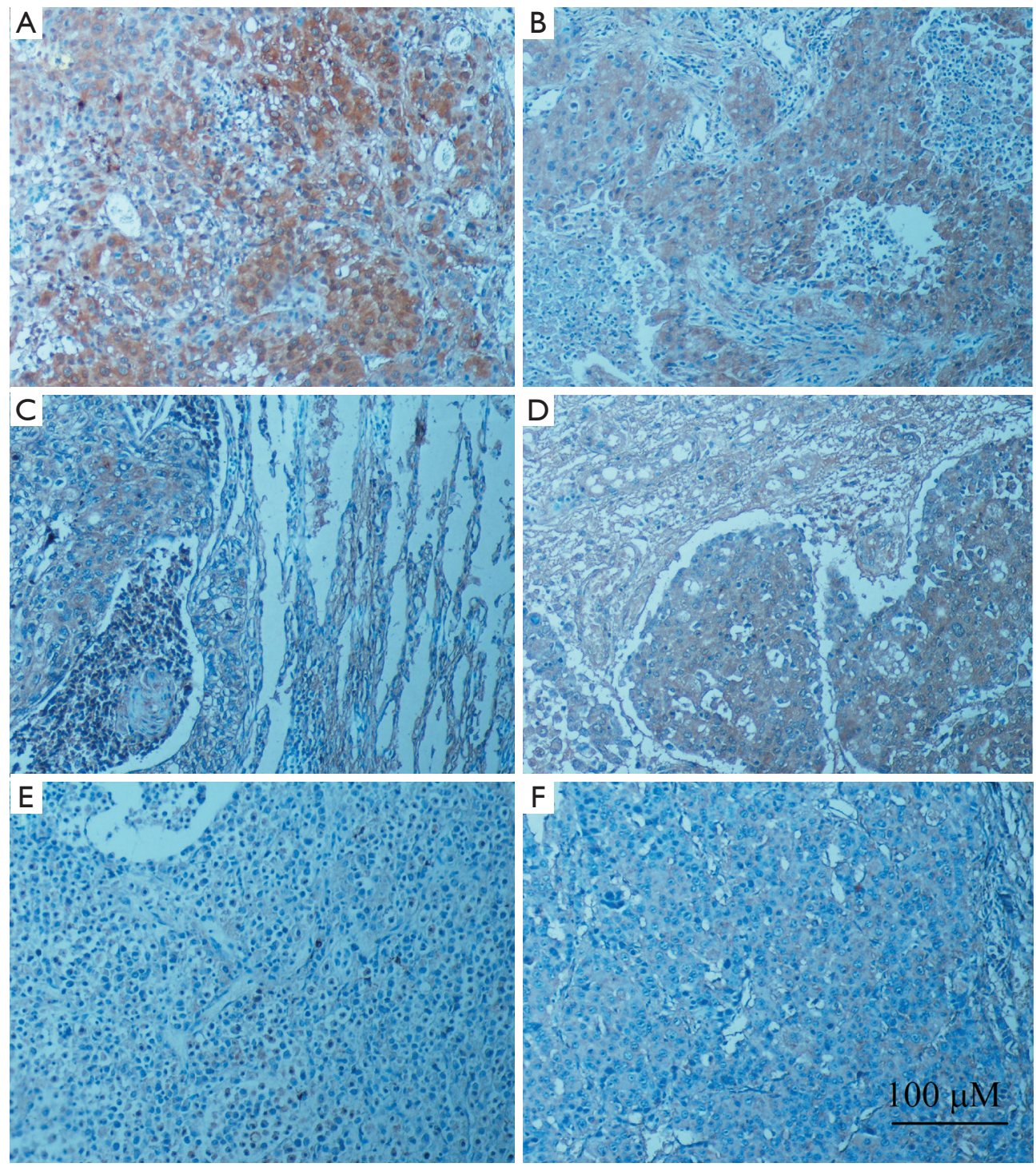

Figure 1 Representative immunohistological images showing different expression intensities of PD-L1 in (A,B,C): pulmonary primary tumors and in (D,E,F): brain metastatic tumors. PD-L1 expression intensity was scored as ++ in (A), as + in (B) and negative in (C). PD-L1 expression intensity was scored as ++ in (D), as + in (E) and negative in (F). PD-L1, programmed death-ligand 1.

index was chosen. The whole population was categorized as low NLR group with 6 cases and high NLR group with 5 cases. The ROC curve was shown in Figure 2.

\section{Association between expression of PD-L1 and ORR, DCR, $D F S$, and $O S$}

All patients with low expression of PD-L1 in PPT achieved PR and CR with $100 \%$ DCR and ORR. The
DCR and ORR in eight patients with high expression of PD-L1 was $87.5 \%$ and $75 \%$ respectively. The median OS in patients with low expression of PD-L1 was 38 and 17 months in those with high expression (Log-Rank test, $\mathrm{P}=0.201$ ). One case with low expression of PD-L1 in PPT was not observed recurrence or progression in PPT lesions during follow up and reached 36 months of OS. Eight patients with low expression of PD-L1 in BM lesions had $62.5 \%$ ORR, $75 \%$ DCR and 7 months of 
DFSbrain. Whereas three patients with high expression of PD-L1 in BM lesions had 66.7\% ORR and DCR with only 3 months of DFSbrain. As to OS, Eight patients with low expression of PD-L1 in BM lesions had 27 months of median OS, which was significantly longer than patients with high expression of PD-L1 with 10 months of median OS $(\mathrm{P}=0.012)$ (Figure $3 A)$. Six patients with consistent expression of PD-L in both PPT and BM lesions had 36 months of median OS and only 17 months mOS was observed in rest patients $(\mathrm{P}=0.104)$ (Tables 2,3). This result could be attributed to fact that $83.8 \%$ patients had low expression in both PPT and BM lesions. Patients with low expression of PD-L1 was featured with early-stage, those with high expression was all classified III stage or higher.

\section{Association between NLR in peripheral blood prior BM lesions surgery and ORR, DCR, DFS as well as OS}

The cutoff value of NLR in peripheral blood prior BM lesions surgery based on Youden index was fixed on 3.0553, which categorized whole population into high NLR group with 5 cases $(45.5 \%)$ and low NLR group with 6 cases (54.5\%). In high NLR group, 4 patients had reached CR or PR with $80 \%$ ORR and $80 \%$ DCR. Six patients in low NLR group had all reached disease control with $100 \%$ DCR, in which 5 patients had CR or PR exhibited $83.8 \%$ ORR being slightly higher than high NLR group. DFSbrain was 3 months (95\% CI: 0.583-5.147 months) in high NLR group, whereas the low NLR group had 7 months of DFSbrain (95\% CI: 3.399-10.601, LogRank $\mathrm{P}=0.724)$. However, the OS in low NLR group was borderline significantly longer than high NLR group (mOS: 27 vs. 10, Log-Rank $\mathrm{P}=0.091$ ) (Figure 3B). One patient with lowest NLR had 9 months of DFSbrain and 38 months of mOS, in contrast, the patient with highest NLR had merely 3 months of DFSbrain and 6 months of mOS (Tables 4,5).

\section{Combined PD-L1 and NLR to predict DFS and OS}

To explore more efficient prognostic biomarker, NLR in peripheral blood prior BM lesions surgery and expression of PD-L1 in BM lesions were combined to evaluate performance. Amongst 8 patients with low expression of PD-L1 in BM lesions, 5 patients also exhibited low NLR (less than cutoff value of 3.0553 ), other 3 patients had high NLR. Low expression of PD-L1 in BM lesions was observed in 3 patients which consisted of only one patient with low NLR and two patient with high NLR. There were 5 patients with both low expression of PD-L1 and low NLR who had 9 months of DFSbrain profoundly longer than others who had only 3 months of DFSbrain (LogRank, $\mathrm{P}=0.455)$, meanwhile, these 5 patients demonstrated about $60 \%$ reduced progression risk compared with others ( $\mathrm{HR}=0.600,95 \%$ CI: 0.437-6.348, $\mathrm{P}=0.455$ ). Furthermore, these 5 patients reached 36 months of mOS significantly longer than others with only 10 months of mOS (Log-Rank, $\mathrm{P}=0.034, \mathrm{HR}=0.133$, 95\% CI: 0.861-65.819, $\mathrm{P}=0.068$ ) (Figure 3C, Table 6).

\section{Discussion}

Non-small cell lung cancer patients who have had brain metastasis usually possesses extreme poor prognosis with OS about 2-4 months (1). However, traditional treatments such as chemotherapy, whole-brain radiotherapy (WBRT), and stereotactic radiotherapy and even surgery to reset single lesion are all still disappointed for cure and improvement of survivals. Accordingly, many efforts have been focused on novel therapeutic targets and strategies to improve clinical outcomes of NSCLC with brain metastasis and utilization of immune checkpoint inhibitors against PD-/PD-L1 pathway is becoming attractive field.

Both PD-1 and PD-L1 are immunosuppressive factors, and activation of the PD-1/PD-L1 pathway leads to the formation of immunosuppressive tumor microenvironment that allows tumor cells to escape immune surveillance and killing (3). Overexpression of PD-1 or/and PD-L1 was observed in many malignant tumors including NSCLC $(5,6)$, breast cancer (21) and melanoma (22). The immune checkpoint inhibitor against PD-1/PD-L1 in advanced NSCLC showed remarkable curative effect both in the first and the second line treatment than chemotherapy. Moreover, the expression and the cellular distribution alteration of PD-L1 can be used as biological indicators of tumor invasion, the sensitivity to radiotherapy or chemotherapy and prognosis. But many of clinicopathologic characteristics including staging, pathological types, genomic mutations, expression of PD-L1 and therapeutic variables such as drug dosage, frequency, and combination with other chemotherapy or targeted therapy drugs could potentially influence response to immune checkpoint inhibitor against PD-1/PD-L1 in clinical practice. Therefore, like targeted therapeutic, it is very crucial to find the most powerful predictive biomarkers for the clinical precise treatment with PD-L1 inhibitors.

Recent studies have shown that the occurrence 
and development of malignant tumors are related to inflammatory response. Namely, tissue destruction caused by tumor infiltration leads to non-specific inflammatory reaction and abnormal phenotype of tumor cells also can promote inflammatory cells infiltration $(23,24)$. The cytokines and other inflammatory mediators secreted by neutrophil granulocytes in tumor microenvironment provide

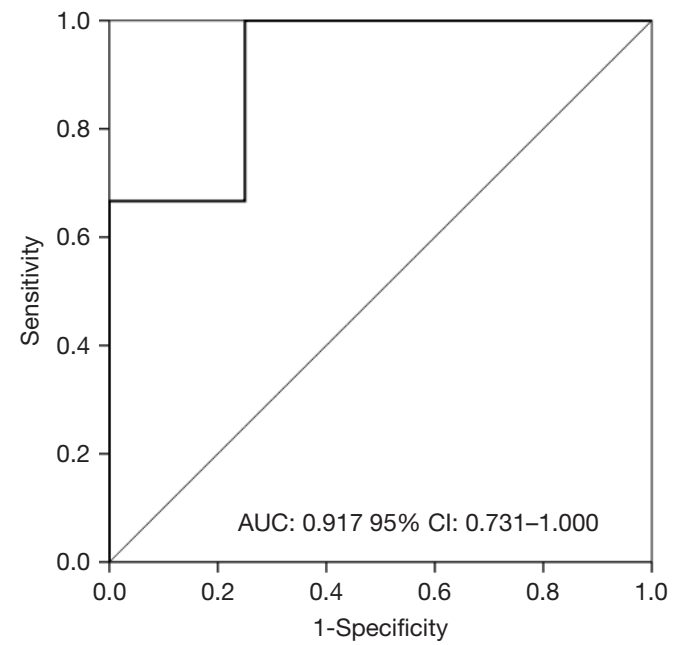

Figure 2 ROC curve showing discrimination of patients reaching disease control from others based on the neutrophil-to-lymphocyte ratio in peripheral venous blood before brain surgery. ROC, receiver operating characteristic. good conditions for the growth and reproduction of tumor cells. Besides, neutrophil granulocytes can produce vascular endothelial growth factor to stimulate angiogenesis to promote tumor growth and invasion (25). Lymphocytes are mainly responsible for tumor-specific immune responses, especially $\mathrm{CD} 3+\mathrm{T}$ cells and natural killer cells which play an important role in the process of induced damage and apoptosis of tumor cells. T lymphocytes are also enabled to secrete cytokines to inhibit proliferation and metastasis of tumor cells (26). The decline in the number of lymphocytes indicates the abnormal immune system which could reduce the immunity to tumor. Many shreds of evidence provided the concept that when tumor cells grow rapidly to transcend the limitation of host antitumor immunity, the tumor cells can be adapted to exhibit immune tolerance and logarithmic reproduction and lead to micrometastasis (27).

In addition, the decrease of lymphocytes in the adjacent tissues is also beneficial to the invasion and development of tumor tissue. Therefore, increase of the neutrophil granulocytes and accompanying a decrease of lymphocytes in peripheral blood which leads to high value of NLR is usually observed in advanced cancers (24). Hirashima (28) and Gomez (29) found that patients with high NLR were often associated with decreased lymphocytes or natural killer cell activity, leading to dysfunction of lymphocytemediated tumor immune. In addition, an elevated preoperative NLR in both blood and tissue has been shown to negatively correlate with the prognosis of patients with
A

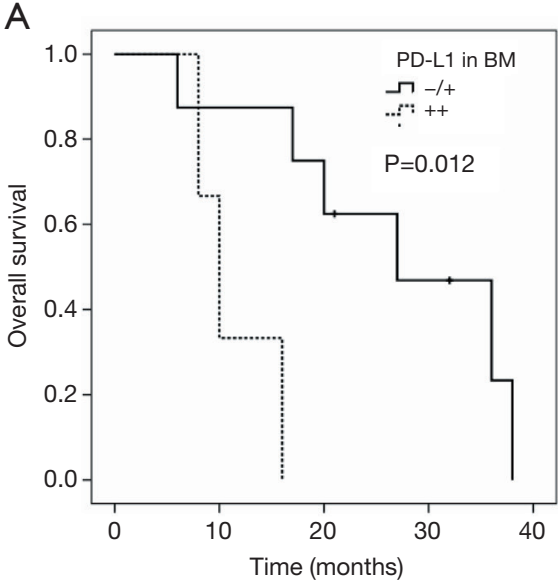

B

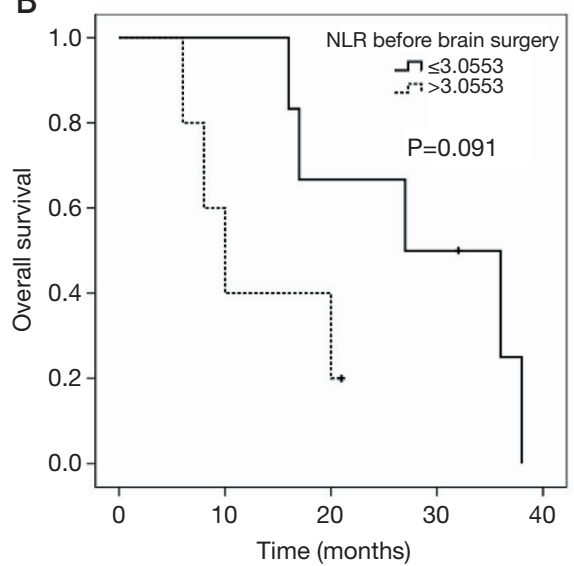

C

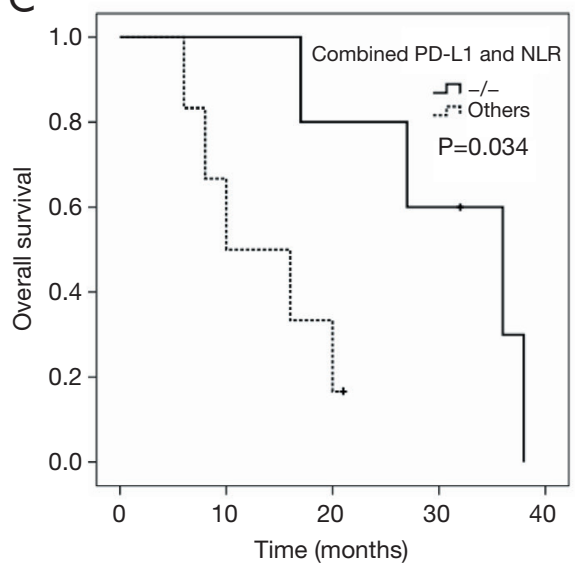

Figure 3 Kaplan-Meier curves illustrating superior survival in patients with (A) low brain PD-L1 expression, (B) low neutrophil to lymphocyte ratio before brain surgery and (C) both low PD-L1 expression and low neutrophil to lymphocyte ratio. Symbol -/- denotes both low PD-L1 expression in brain metastasis tumors and low NLR value. PD-L1, programmed death-ligand 1; PPT, pulmonary primary tumor; BM, brain metastasis; NLR, neutrophil to lymphocyte ratio. 
Table 2 Association between PD-L1 expression in PPT and BM and ORR, DCR

\begin{tabular}{|c|c|c|c|c|c|}
\hline PD-L1 & $\mathrm{N}=11(\%)$ & \multicolumn{2}{|c|}{ ORR (CR + PR) } & \multicolumn{2}{|c|}{$\mathrm{DCR}(\mathrm{CR}+\mathrm{PR}+\mathrm{SD})$} \\
\hline PD-L1 in PPT & & & 1.000 & & 1.000 \\
\hline Low & $3(27.3)$ & $3(100.0)$ & & $3(100.0)$ & \\
\hline High & $8(72.7)$ & $6(75.0)$ & & 7 (87.5) & \\
\hline Low & $8(72.7)$ & $5(62.5)$ & & $6(75.0)$ & \\
\hline High & $3(27.3)$ & $2(66.7)$ & & $2(66.7)$ & \\
\hline \multicolumn{6}{|c|}{ Consistency in PPT and BM } \\
\hline Inconsistency & $5(45.5)$ & - & - & - & - \\
\hline
\end{tabular}

PPT, pulmonary primary tumor; BM, brain metastasis; ORR, objective response rate; CR, complete response; PR, partial response; DCR, disease control rate; SD, stable disease.

Table 3 Association between PD-L1 expression in PPT and BM and DFS, OS

\begin{tabular}{|c|c|c|c|c|c|}
\hline PD-L1 & $\mathrm{N}=11(\%)$ & \multicolumn{2}{|c|}{ DFS (months) } & \multicolumn{2}{|c|}{ OS (months) } \\
\hline PD-L1 in PPT & & & 0.800 & & 0.201 \\
\hline Low & $3(27.3)$ & 8 & & 38 & \\
\hline High & $8(72.7)$ & $12(0.301-23.699)$ & & $17(11.456-22.544)$ & \\
\hline Low & $8(72.7)$ & $7(2.842-11.158)$ & & 27 (12.026-41.974) & \\
\hline High & $3(27.3)$ & $3(0-6.201)$ & & $10(6.799-13.201)$ & \\
\hline Consistency in PPT and BM & & & - & & 0.104 \\
\hline Inconsistency & $5(45.5)$ & - & & $36(0-74.921)$ & \\
\hline
\end{tabular}

PPT, pulmonary primary tumor; BM, brain metastasis; ORR, objective response rate; DCR, disease control rate; OS, overall survival; DFS, disease-free survival.

HCC $(30,31)$. At the same time, detection of preoperative blood cell classification and counting is used in clinical routine application with the advantages of being easy to operate and economical. So, NLR index is relatively easy to obtain and has considerable specificity and sensitivity. Therefore, NLR has attracted much attention as a new biomarker for the evaluation of the prognosis of solid tumors (12-14).

This study was a retrospective study of small samples of 11 patients who had received surgery of lung lesions of primary tumor and brain metastasis in NSCLC patients with BM. The association of ORR, DCR, DFS, and OS with expression pattern of PD-L1 in primary lung tumor and BM and NLR prior surgery were systemically analyzed in details. From our preliminary results, OS was significantly longer in patients with low PD-L1 expression in $\mathrm{BM}$ lesions than those with high expression of PD-L1 (mOS: 27 vs. 10 months, $\mathrm{P}=0.012$ ). Patients with clinicopathologic characteristics such as squamous cell carcinoma, female, never smokers, solitary BM were 
Table 4 Association between NLR before metastatic brain lesion surgery and ORR, DCR

\begin{tabular}{|c|c|c|c|c|c|}
\hline NLR before brain surgery & $\mathrm{N}=11(\%)$ & \multicolumn{2}{|c|}{ ORR (CR + PR) } & \multicolumn{2}{|c|}{$\mathrm{DCR}(\mathrm{CR}+\mathrm{PR}+\mathrm{SD})$} \\
\hline$>3.0553$ & $5(45.5)$ & $4(80.0)$ & \multirow{2}{*}{1.000} & $4(80.0)$ & \multirow{2}{*}{0.455} \\
\hline$\leq 3.0553$ & $6(54.5)$ & $5(83.3)$ & & $6(100.0)$ & \\
\hline
\end{tabular}

NLR, neutrophil-to-lymphocyte ratio; ORR, objective response rate; CR, complete response; PR, partial response; DCR, disease control rate; SD, stable disease.

Table 5 Association between NLR before metastatic brain lesion surgery and DFS, OS

\begin{tabular}{|c|c|c|c|c|c|}
\hline NLR before brain surgery & $\mathrm{N}(\%)$ & \multicolumn{2}{|c|}{ DFS (months) } & \multicolumn{2}{|c|}{ OS (months) } \\
\hline$\leq 3.0553$ & $6(54.5)$ & 7 (3.399-10.601) & 0.724 & 27 (8.756-45.244) & 0.091 \\
\hline
\end{tabular}

NLR, neutrophil-to-lymphocyte ratio; OS, overall survival; DFS, disease-free survival.

Table 6 Analysis of combined PD-L1 expression and NLR before brain surgery for DFS and OS

\begin{tabular}{|c|c|c|c|c|}
\hline NLR before brain surgery & \multicolumn{2}{|c|}{ DFSbrain (months) } & \multicolumn{2}{|c|}{ OS (months) } \\
\hline$-1-$ & $9(4.706-13.294)$ & \multirow{2}{*}{0.432} & 36 (21.962-50.038) & \multirow{2}{*}{0.034} \\
\hline Others & $3(1.491-4.509)$ & & 10 (0.398-19.602) & \\
\hline
\end{tabular}

-/-, low expression of PD-L1 and low NLR (less than cutoff value of 3.0553). NLR, neutrophil-to-lymphocyte ratio; OS, overall survival;

DFS, disease-free survival.

more sensitive to treatment and exhibited higher local control and better prognosis. Compared with patients who received chemotherapy alone, patients who received targeted therapy had higher efficiency. These results of our study were in consistence with IPASS (32) and ECOG4599 and AVAil $(33,34)$ which reported that for patients with EGFR mutations, the ORR was increased by $20 \%$ in patients treated with TKI drugs compared with those with the platinum-based regimens. Furthermore, in addition to the higher response rate, patients who received the targeted therapy reached 26 months of median DFSlung which is increased by 18 months compared with patients who received chemotherapy alone. However, progression-free survival regarding intracranial lesions was no difference between these two groups. It is speculated that both chemotherapeutic drugs and TKIs drugs do not effectively penetrate the brain metastasis through bloodbrain barrier resulting in low drug concentration. The result that final benefit concerning OS is from longer DFS
(16 vs. 26 months, $\mathrm{P}=0.608$ ) was consistent with results from NEJGSG002 and OPTIMAL trials in which the firstgeneration EGFR-TKIs drugs had shown an important role in promoting OS in advanced NSCLC patients harboring EGFR sensitive mutations (32-37). The results of multivariate analysis of DFS and OS showed the expression of PD-L1 in lung lesions, NLR prior surgery, gender, age, smoking status, histological type, T stage, PS score and brain metastasis time were all not significantly associated with DFS and OS. Only the expression of PD-L1 in brain metastatic lesion was the independent prognostic factors for OS, high expression of PD-L1 was relevant with a worse prognosis.

The expression pattern of PD-L1 in primary lung lesions and brain metastasis lesions in 11 patients showed cytoplasmic expression. It was found that the high expression of PD-L1 is associated with resistance to radiotherapy or chemotherapy and poor prognosis. Meanwhile, for patients had BM occurred during the 
treatment process, there was a significant correlation between the expression intensity of PD-L1 in primary lung tumor and time of brain metastasis merged: the higher expression intensity of PD-L1, the more prone to brain metastasis, which indicated that overexpression of PDL1 promotes invasion and metastasis. The correlation between PD-L1 expression and DFS and OS was the main purposes of this research, regardless of pulmonary primary or brain metastatic tumors, higher expression of PD-L1 was always associated with shorter DFS and more prone to local recurrence; But PD-L1 expression in brain metastasis lesions was an only independent prognostic factor for OS. It was worth noting that there is a case with negative expression of PD-L1 in primary lung lesions and $\mathrm{BM}$. The lung lesions of this patient remained stable during the whole period of follow up. The distant metastasis led to the treatment failure, with final 39 months of OS. Taken together, the above results indicated that the higher expression of PD-L1 caused more invasiveness of cancer cells, more resistance to treatment and the worse prognosis. In addition, we also found that the relationship between the expression of PD-L1 and T, N stage, the low expression of PD-L1 was found enriched in patients with earlier $\mathrm{T}$ and $\mathrm{N}$ stage, whereas, patients with late-stage $\mathrm{T} 4$ or N2, clinical stage III or more were found to have the high expression of PD-L1. These results revealed close relationship between the invasiveness and prognosis of tumor and the expression intensity of PD-L1: the low expression is always associated with the earlier $\mathrm{T}$ stage, less lymph node metastasis and good prognosis, on the contrary, the high expression is associated with poor prognostic factors.

Combined analysis of expression pattern of PD-L1 in paired primary lung lesions and BM showed that PD-L1 expression is not consistent in 5 patients with BM. Patients with consistent expression had significant longer OS than those inconsistent in expression of PD-L1 (36 vs. 17 months, $\mathrm{P}=0.104)$. The explanation to this may rely on the fact that $83.3 \%$ of the patients with consistent expression are low expression of PD-L1 which leads to the less immune escape and the better efficacy and prognosis. Some studies $(38,39)$ reported patients with brain metastasis have higher incidence of primary lung tumor EGFR mutation. Recent study (40) revealed that 70 single nucleotide variants (SNVs) detected in the primary tumor is lost and other $83 \mathrm{SNVs}$. are merged in metastatic tumor through 1,264 cancerrelated genes sequencing in 47 primary and metastatic tumor samples from 21 cases of colorectal cancer. These 83 new mutations play an important role in several key pathways which are more appropriate for selectin of patients with corresponding targeted therapy, and the metastatic tumor is more faithful to reflect tumor genome. In conclusion, the genetic variations between the primary and metastatic tumor are inconsistent. Therefore, it is inadequate using the expression of one gene in the lung primary tumor to predict the status of the same gene in brain metastatic lesion or therapeutic response. At present, reconstructing subclonal composition and evolution of brain metastasis in NSCLC patients is worthy of further study.

In our study, the low NLR group had much longer OS than the high NLR group (27 vs. 10 months, $\mathrm{P}=0.091$ ). This may be due to the release of proangiogenic factors by neutrophils which can further stimulate tumor angiogenesis contributing to tumor progression. At the same time, increased neutrophils caused depression of lymphocytes resulting in decreased antitumor immune response. The systemic inflammatory response provided further condition to promote tumor development and poor prognosis. This is consistent with other reports in lung cancer (41-43).

Both PD-L1 and NLR represents the immune and inflammatory state of the human body and can affect each other. There was evidence that PD-L1 was overexpressed on tumor cells (44), Kupffer cells (45) and TAMs (46), which induced apoptosis of $\mathrm{T}$ cells and immune tolerance in the case of HCC. The interruption of the interaction between PD-L1 and PD-1 could be able to reduce cancer development and enhance the $\mathrm{T}$ cell-mediated antitumor activity. Taken together, PD-L1/PD-1 signaling pathway had an important role in the development of HCC. Whereas, Neutrophils have been considered nonprofessional antigen-presenting cells (APCs). Recent studies have demonstrated the induced expression of co-inhibitory molecules, such as PD-L1, following in vitro exposure to cytokines (47) or after stimulation by LPS or toll-like receptors (TLRs) (48). PD-L1 expression on neutrophils was also observed to be increased in vivo in patients with active tuberculosis (TB) or HIV infection while it was decreased in patients who received anti-TB therapy (49) or anti-viral treatment (48). These reports suggested the effect of PD-L1 expression on neutrophils function. A combined analysis revealed that patients with low NLR were found in $62.5 \%$ of the patients with low expression of PD-L1, and the patients with high NLR value in $66.7 \%$ of high expression of PD-L1 which indicated there is a correlation between the expression level of PD-L1 and NLR. High expression of PD-L1 was always accompanied by higher NLR and had more invasive behavior and poor prognosis. 
The patients with both low expression of PD-L1 and low NLR are much superior to the rest of the patients regarding response to treatment and prognosis. This is also consistent with the conclusion reported by $\mathrm{Ha}$ et al. that high SPDL1 and high NLR is independent poor prognostic factors in the advanced BTC (50). A retrospective study of 58 patients with advanced NSCLC found that patients with $\geq 4$ NLR before immune checkpoint inhibitors against PD-L1 treatment possess shorter OS and disease progression (DFS: 11.4 vs. 10.1 months; OS: NR vs. 19.5 months). NLR may be a predictor for the efficacy of PD-1/PD-L1 antibody treatment. Although no patients received PD-L1 treatment in our study, the fact that NLR and PD-L1 have obvious correlation highly suggests that the value of preoperative NLR can not only predict therapeutic response and prognosis but also can be used as biomarkers for predicting the efficacy of PD-L1 treatment.

Taken together, regardless of the primary lung lesion or $\mathrm{BM}$ lesions, the higher expression of PD-L1 is associated with worse prognosis, while patients with low PD-L1 expression have prolonged survival. Deteriorated response to treatment is mainly observed in patients with high preoperative NLR value. Both high expression of PD-L1 and high value of preoperative NLR value indicate more aggressive malignant biological behavior, whereas both low values indicate good prognosis.

In conclusion, our study demonstrated that the preoperative PD-L1 expression and NLR value is associated with response and prognosis in advanced NSCLC. Both high expression of PD-L1 and high NLR value confers less sensitivity to treatment and associated with poor prognosis. The limitation of this research is rather limited sample size which needs to be further verified by expanding the sample size. Since the PD-L1 antibody has not yet been marketed in China, all patients in this study did not receive anti-PD-L1 treatment. The prospective studies should be conducted after PD-L1 listing to further validate the findings of this study. Besides, functional molecular variations conferring to response to immune checkpoint inhibitors should be also investigated to provide a theoretical fundamental for personalized therapy in the future.

\section{Acknowledgments}

The authors thank Mr. Xian-Feng Lu and Miss. Mao-Jun Liao for their kind and excellent technical assistance.

Funding: This study was supported by Grants from the Youth Innovation Foundation of Institute (No.
2014YQN02) to L Xia.

\section{Footnote}

Conflicts of Interest: All authors have completed the ICMJE uniform disclosure form (available at http://dx.doi. org/10.21037/tcr.2019.11.08). The authors have no conflicts of interest to declare.

Ethical Statement: The authors are accountable for all aspects of the work in ensuring that questions related to the accuracy or integrity of any part of the work are appropriately investigated and resolved. This study was conducted rigorously according to the Declaration of Helsinki and the principle of International Ethical Guidelines for Biomedical Research Involving Human constituted by the World Health Organization (WHO) and the Council for International Organizations of Medical Sciences (CIOMS) and was approved by the Ethics Committee of the Daping Hospital. The written consensus was obtained from all participants.

Open Access Statement: This is an Open Access article distributed in accordance with the Creative Commons Attribution-NonCommercial-NoDerivs 4.0 International License (CC BY-NC-ND 4.0), which permits the noncommercial replication and distribution of the article with the strict proviso that no changes or edits are made and the original work is properly cited (including links to both the formal publication through the relevant DOI and the license). See: https://creativecommons.org/licenses/by-nc-nd/4.0/.

\section{References}

1. Yawn BP, Wollan PC, Schroeder C, et al. Temporal and gender-related trends in brain metastases from lung and breast cancer. Minn Med 2003;86:32-7.

2. Khuntia D, Brown P, Li J, et al. Whole-brain radiotherapy in the management of brain metastasis. J Clin Oncol 2006;24:1295-304.

3. Pardoll DM. The blockade of immune checkpoints in cancer immunotherapy. Nat Rev Cancer 2012;12:252-64.

4. Keir ME, Butte MJ, Freeman GJ, et al. PD-1 and its ligands in tolerance and immunity. Annu Rev Immunol 2008;26:677-704.

5. Velcheti V, Schalper KA, Carvajal DE, et al. Programmed death ligand-1 expression in non-small cell lung cancer. Lab Invest 2014;94:107-16. 
6. Boland JM, Kwon ED, Harrington SM, et al. Tumor B7$\mathrm{H} 1$ and $\mathrm{B} 7-\mathrm{H} 3$ expression in squamous cell carcinoma of the lung. Clin Lung Cancer 2013;14:157-63.

7. Gatalica Z, Snyder C, Maney T, et al. Programmed cell death 1 (PD-1) and its ligand (PD-L1) in common cancers and their correlation with molecular cancer type. Cancer Epidemiol Biomarkers Prev 2014;23:2965-70.

8. Azuma K, Ota K, Kawahara A, et al. Association of PDL1 overexpression with activating EGFR mutations in surgically resected non-small-cell lung cancer. Ann Oncol 2014;25:1935-40.

9. Zhou ZJ, Zhan P, Song Y. PD-L1 over-expression and survival in patients with non-small cell lung cancer: a meta-analysis. Transl Lung Cancer Res 2015;4:203-8.

10. Tang $Y$, Fang $W$, Zhang $Y$, et al. The association between PD-L1 and EGFR status and the prognostic value of PDL1 in advanced non-small cell lung cancer patients treated with EGFR-TKIs. Oncotarget 2015;6:14209-19.

11. Coussens LM, Werb Z. Inflammation and cancer. Nature 2002;420:860-7.

12. Azab B, Bhatt V R, Phookan J, et al. Usefulness of the neutrophil-to-lymphocyte ratio in predicting short-and long-term mortality in breast cancer patients. Ann Surg Oncol 2012;19:217-24.

13. Pichler M, Hutterer G, Stoeckigtc C, et al. Validation of the pre-treatment neutrophil-lymphocyte ratio as a prognostic factor in a large European cohort of renal cell carcinoma patients.Br J Cancer 2013;108:901-7.

14. Stotz M, Gerger A, Eisner F, et al. Increased neutrophillymphocyte ratio is a poor prognostic factor in patients with primary operable and inoperable pancreatic cancer. Br J Cancer 2013; 109:416-21.

15. Lieto E, Galizia G, Auricchio A, et al. Preoperative Neutrophil to Lymphocyte Ratio and Lymphocyte to Monocyte Ratio are Prognostic Factors in Gastric Cancers Undergoing Surgery. J Gastrointest Surg 2017;21:1764-74.

16. Scilla KA, Bentzen SM, Lam VK, et al. NeutrophilLymphocyte Ratio is a Prognostic Marker in Patients with Locally Advanced (Stage IIIA and IIIB)Non-Small Cell Lung Cancer Treated with Combined Modality Therapy. Oncologist 2017;22:737-42.

17. Kim JH, Lee JY, Kim HK, et al. Prognostic significance of the neutrophil-to-lymphocyte ratio and plateletto-lymphocyte ratio in patients with stage III and IV colorectal cancer. World J Gastroenterol 2017,23:505-15.

18. Postmus PE, Brambilla E, Chansky K, et al. The IASLC Lung Cancer Staging Project: proposals for revision of the $M$ descriptors in the forthcoming (seventh) edition of the TNM classification of lung cancer. J Thorac Oncol 2007;2:686-93.

19. Groome PA, Bolejack V, Crowley JJ, et al. The IASLC Lung Cancer Staging Project: validation of the proposals for revision of the $T, N$, and $M$ descriptors and consequent stage groupings in the forthcoming (seventh) edition of the TNM classification of malignant tumours. J Thorac Oncol 2007;2:694-705.

20. Eisenhauer EA, Therasse P, Bogaerts J, et al. New response evaluation criteria in solid tumors: revised RECIST guideline (version 1.1). European Journal of Cancer 2009; 45:228-47.

21. Bae SB, Cho HD, Oh MH, et al. Expression of programmed death receptor ligand-1 with high tumorinfiltrating lymphocytes is associated with better prognosis in breast cancer. J Breast Cancer 2016;19:242-51.

22. Spranger S, Spaapen RM, Zha Y, et al. Up-regulation of PD-L1, IDO, and T(regs) in the melanoma tumor microenvironment is driven by CD8(+) T cells. Sci Transl Med 2013;5:200-16.

23. Baniyash M, Sade-Feldman M, Kanterman J. Chronic inflammation and cancer: suppressing the suppressors. Cancer Immunol Immunother 2014;63:11-20.

24. Mantovani A, Allavena P, Sica A, et al. Cancer- related inflammation. Nature 2008;454:436-44.

25. Gregory AD, Houghton AM. Tumor-associated neutrophils: new targets for cancer therapy. Cancer Res 2011;71:2411-6.

26. Ohtani H. Focus on TILs: prognostic significance of tumor-infiltrating lymphocytes in human colorectal cancer. Cancer Immun 2007;7:4.

27. Young AL, Malik HZ, Abu-Hilal M, et al. Large hepatocellular carcinoma: time to stop preoperative biopsy. J Am Coll Surg 2007;205:453-62.

28. Hirashima M, Higuchi S, Sakamoto K, et al. The ratio of neutrophils to lymphocytes and the phenotypes of neutrophils in patients with early gastric cancer. J Cancer Res Clin Oncol 1998;124:329-34.

29. Gomez D, MorNs-Stiff G, Toogood GJ, et al. Impact of systemic inflammation on outcome following resection for intrahepatic cholangiocarcinoma. J Surg Oncol 2008;97:513-8.

30. Mano Y, Shirabe K, Yamashita Y, et al. Preoperative neutrophil-to-lymphocyte ratio is a predictor of survival after hepatectomy for hepatocellular carcinoma: a retrospective analysis. Ann Surg 2013;258:301-5.

31. Motomura T, Shirabe K, Mano Y, et al. Neutrophil- 
lymphocyte ratio reflects hepatocellular carcinoma recurrence after liver transplantation via inflammatory microenvironment. J Hepatol 2013;58:58-64.

32. Mok TS, Wu YL, Thongprasert S, et al. Gefitinib or carboplatin-paclitaxel in pulmonary adenocarcinoma. $\mathrm{N}$ Engl J Med 2009;361:947-57.

33. Sandler A, Gray R, Perry MC, et al. Paclitaxel-carboplatin alone or with bevacizumab for non-small-cell lung cancer. N Engl J Med 2006;355:2542-50.

34. Reck M, von Pawel J, Zatloukal P, et al. Phase III trial of cisplatin plus gemcitabine with either placebo or bevacizumab as first-line therapy for nonsquamous non-small-cell lung cancer: AVAil. J Clin Oncol 2009;27:1227-34.

35. Yi HG, Kim HJ, Kim YJ, et al. Epidermal growth factor receptor (EGFR) tyrosine kinase inhibitors (TKIs) are effective for leptomeningeal metastasis from non-small cell lung cancer patients with sensitive EGFR mutation or other predictive factors of good response for EGFR TKI. Lung Cancer 2009;65:80-4.

36. Mitsudomi T, Morita S, Yatabe Y, et al. Gefitinib versus cisplatin plus docetaxel in patients with non-small-cell lung cancer harboring mutations of the epidermal growth factor receptor (WJTOG3405): an open-label, randomized phase 3 trial. Lancet Oncol 2010;11:121-8.

37. Maemondo M, Inoue A, Kobayashi K, et al. Gefitinib or chemotherapy for non-small-cell lung cancer with mutated EGFR. N Engl J Med 2010;362:2380-8.

38. Bartolotti M, Franceschi E, Brandes AA. EGF receptor tyrosine kinase inhibitors in the treatment of brain metastases from non-small-cell lung cancer. Expert Rev Anticancer Ther 2012;12:1429-35.

39. Zhang J, Yu J, Sun X, et al. Epidermal growth factor receptor tyrosine kinase inhibitors in the treatment of central nerve system metastases from non-small cell lung cancer. Cancer Lett 2014;351:6-12.

40. Kloosterman WP, Hoogstraat M, Paling O, et al. Chromothripsis is a common mechanism driving genomic rearrangements in primary and metastatic colorectal cancer. Genome Biol 2011;12:R103.

Cite this article as: Xia L, Huang $\mathrm{H}$, Xiao H, Wang D, Yang Z. Utilization of combined PD-L1 expression and neutrophil-tolymphocyte ratio prior to surgery as a prognostic factor in nonsmall cell lung cancer with brain metastasis. Transl Cancer Res 2019;8(8):2864-2877. doi: 10.21037/tcr.2019.11.08
41. Wu G, Yao Y, Bai C, et al. Combination of platelet to lymphocyte ratio and neutrophil to lymphocyte ratio is a useful prognostic factor in advanced non-small cell lung cancer patients. Thoracic Cancer 2015;6:275-87.

42. Cannon NA, Meyer J, Iyengar P, et al. Neutrophillymphocyte and platelet-lymphocyte ratios as prognostic factors after stereotactic radiation therapy for earlystage non-small-cell lung cancer. J Thorac Oncol 2015;10:280-5.

43. Kang MH, Go SI, Song HN, et al. The prognostic impact of the neutrophil-to-lymphocyte ratio in patients with small-cell lung cancer. Br J Cancer 2014;111:452-60.

44. Gao Q, Wang XY, Qiu SJ, et al. Overexpression of PDL1 significantly associates with tumor aggressiveness and postoperative recurrence in human hepatocellular carcinoma. Clin Cancer Res 2009;15:971-9.

45. Wu K, Kryczek I, Chen L, et al. Kupffer cell suppression of CD8+ T cells in human hepatocellular carcinoma are mediated by B7-H1/programmed death-1 interactions. Cancer Res 2009;69:8067-75.

46. Kuang DM, Zhao Q, Peng C, et al. Activated monocytes in peritumoral stroma of hepatocellular carcinoma foster immune privilege and disease progression through PD-L1. J Exp Med 2009;206:1327-37.

47. Bankey PE, Banerjee S, Zucchiatti A, et al. Cytokineinduced expression of programmed death ligands in human neutrophils. Immunol Lett 2010;129:100-7.

48. Bowers NL, Helton ES, Huijbregts RP, et al. Immune suppression by neutrophils in HIV-1 infection: role of PD-L1/PD-1 pathway. PLoS Pathog 2014;10:e1003993.

49. McNab FW, Berry MP, Graham CM, et al. Programmed death ligand 1 is over-expressed by neutrophils in the blood of patients with active tuberculosis. Eur J Immunol 2011;41:1941-7.

50. Ha H, Nam AR, Bang JH, et al. Soluble programmed death-ligand 1 (sPDL1) and neutrophil-to-lymphocyte ratio (NLR) predicts survival in advanced biliary tract cancer patients treated with palliative chemotherapy. Oncotarget 2016;7:76604-12. 